\title{
BC-02 eradicates liver cancer stem cells by upregulating the ROS-dependent DNA damage
}

\author{
CHUNHUI DOU ${ }^{1 *}$, CHUNYAN FANG $^{1 *}$, YAN ZHAO ${ }^{1}$, XIAOYAN FU ${ }^{2}$, \\ YUFEI ZHANG ${ }^{1}$, DONGQI ZHU ${ }^{1}$, HUINA WU ${ }^{1}$, HUIJIE LIU ${ }^{1}$, JIAN ZHANG ${ }^{3}$, WENFANG XU ${ }^{4}$, \\ ZHIJUN LIU ${ }^{2}$, HONGYAN WANG ${ }^{2}$, DAQI LI $^{5}$ and XUEJIAN WANG ${ }^{1,6}$
}

${ }^{1}$ Department of Pharmacology, School of Pharmacy, ${ }^{2}$ School of Clinical Medicine, ${ }^{3}$ School of Pharmacy, Weifang Medical
University, Weifang, Shandong 261053; ${ }^{4}$ Department of Medicinal Chemistry, School of Pharmacy, Shandong University,
Jinan, Shandong 250012; ${ }^{5}$ Department of Hematology, Jinan Central Hospital Affiliated to Shandong University, Jinan,
Shandong 250013; ${ }^{6}$ Molecular Oncology Laboratory, Weifang Medical University, Weifang, Shandong 261053 , P.R. China

Received July 7, 2017; Accepted September 20, 2017

DOI: 10.3892/ijo.2017.4159

\begin{abstract}
Cancer stem cells (CSCs) are responsible for chemoresistance, tumor recurrence and metastasis. Reportedly, aminopeptidase N (APN, also known as CD13) is a marker for semi-quiescent CSCs and a therapeutic target in human liver CSCs. In the present study, the effect of BC-02, a compound obtained by conjugating a CD13 inhibitor bestatin and fluorouracil (5-FU), was investigated toward liver CSCs. Tumor spheres formed in serum-free culture conditions have been successfully used to enrich CSCs. In this study, the sphere cells were shown to have several characteristics of CSCs, including drug resistance, high tumorigenicity, epithelial-mesenchymal transition (EMT) phenotype, lower reactive oxygen species (ROS) levels, greater colony-forming efficiency and increased proliferation capacity in vitro. Furthermore, BC-02 effectively suppressed self-renewal and malignant proliferation of CSCs compared with 5-FU, bestatin, and even the combination of 5-FU and bestatin. In addition, cell proliferation was effectively
\end{abstract}

Correspondence to: Professor Xuejian Wang or Professor Chunyan Fang, Department of Pharmacology, School of Pharmacy, Weifang Medical University, Weifang, Shandong 261053, P.R. China E-mail: wangxuejian@wfmc.edu.cn

E-mail: chunyanfang@126.com

*Contributed equally

Abbreviations: CSCs, cancer stem cells; LCSCs, liver cancer stem cells; APN or CD13, aminopeptidase N; 5-FU, fluorouracil; EMT, epithelial-mesenchymal transition; CD13 Ab, CD13-neutralizing antibody; HCC, hepatocellular carcinoma; TICs, tumor-initiating cells; ESI-MS, electrospray ionization mass spectrometry; MTT, methyl thiazolyl tetrazolium; PI, propidium iodide

Key words: cancer stem cells, BC-02, tumor spheres, chemoresistance, CD13, ROS suppressed when exposed to 5-FU plus CD13-neutralizing antibody (CD13 Ab) compared with 5-FU alone. BC-02 can effectively inhibit the activity of CD13. Results demonstrated that CD13 inhibitor BC-02 impaired the properties of liver CSCs by targeting CD13 and upregulating the intracellular ROS and ROS-induced DNA damage. BC-02 might be a potential therapeutic agent for eradicating the liver CSCs and overcoming chemoresistance in liver cancer.

\section{Introduction}

Hepatocellular carcinoma (HCC) is one of the most prevalent malignancies worldwide, and its morbidity and mortality rates have been increasing in recent years $(1,2)$. Currently, systemic therapies and anticancer drugs for HCC have obtained considerable advances; however, the incidence rate of resistance to chemotherapy, tumor recurrence and metastasis is still increasing $(3,4)$. Accumulating evidence has revealed that one of the reasons for the resistance to anticancer drugs is the existence of CSCs or tumor-initiating cells (TICs) (5).

The concept of CSCs or TICs was first proposed approximately 50 years ago (6). The existence of CSCs has been demonstrated in leukemia (7) and solid tumors, including breast, colon, brain and liver cancers (8-13). CSC is a small subpopulation of cancer cells with stem cell properties, such as self-renewal, pluripotency, chemoresistance and limitless proliferation (14). Substantial evidence revealed that CSCs are responsible for initiation and resistance to chemotherapy; and the cancer always relapses and metastasizes due to the persistence of CSCs $(14,15)$. Hence, inhibiting CSC proliferation or even eradicating them may be a pivotal strategy for overcoming chemoresistance and improving the curative effect in liver cancer.

To better investigate CSC properties and novel anticancer drugs, many studies have been created in vitro model system using serum-free stem cell conditional medium to select and expand CSCs. Tumor spheres formed in this condition possess cancer stem cell characteristics, including self-renewal, proliferation, chemoresistance and higher tumorigenicity. Thus, they are considered to be CSCs $(16,17)$. This sphere cell 
A<smiles>CC(C)C[C@H](NC(=O)C(O)[C@H](N)Cc1ccccc1)C(=O)O</smiles><smiles>O=c1[nH]cc(F)c(=O)[nH]1</smiles>

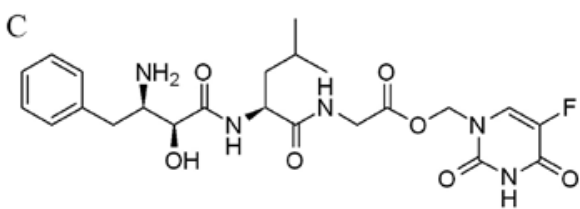

Figure 1. Chemical structures of bestatin (A), 5-FU (B) and the compound BC-02 (C)

formation method has been successfully used to enrich CSCs from liver, brain, lung and ovarian cancers (17-20). Thus, this method was also used to enrich CSCs, and the condition of the culture medium was optimized to create a more suitable microenvironment for better forming liver cancer stem cells (LCSCs).

CD13/APN is a marker for semi-quiescent CSCs and a therapeutic target in human liver CSCs (5). CD13 is a zincbinding type 2 transmembrane ectopeptidase $(150 \mathrm{kDa})$, which is related to malignant behavior in many cancers, such as prostate, colon and lung cancers (21-23). In in vivo transplantation model of mice, $\mathrm{CD}_{13}{ }^{+}$cells survived, and the expression of CD13 are upregulated after 5-FU treatment alone, which is one of the most common cytotoxic drugs in HCC treatment. Thus, co-processing with a CD13 inhibitor could be helpful for radical elimination of $\mathrm{CD}^{2} 3^{+}$cells or liver CSCs (24). Bestatin (ubenimex) is reported to be a CD13 inhibitor, which is constantly used for adult acute nonlymphocytic leukemia (25). As expected, combining bestatin with 5-FU treatment efficiently elicits tumor regression and improves the effect of liver cancer treatment (5). Thus far, few drugs, which could not only target liver CSCs but has cytotoxic effects on the CSCs, may kill CSCs and reveal a promising cure for liver cancer.

In the present study, the serum-free stem cell conditional medium was optimized and sphere cells were successfully enriched and expanded, which possessed the characteristics of stem cells. The compound BC-02, an APN/CD13 inhibitor, showed a significant effect on self-renewal and malignant proliferation of liver CSCs compared to the control, bestatin, 5-FU and even 5-FU plus bestatin. Subsequently, BC-02 could target CD13 and increase the intracellular ROS and the ROS-induced DNA damage, thereby inhibiting the liver CSCs and overcoming the chemoresistance in liver cancer. Therefore, BC-02 might be a novel therapeutic drug for improving the survival rate of liver cancer patients.

\section{Materials and methods}

Chemicals and reagents. Bestatin (Fig. 1A) and 5-FU (Fig. 1B) were obtained from Shanghai Biochempartner Co., Ltd. (Shanghai, China). The compound BC-02 (Fig. 1C) was synthesized by conjugating a CD13 inhibitor bestatin and 5-FU in the Department of Medicinal Chemistry, School of Pharmacy, Shandong University (26). The BC-02 could be decomposed into 5-FU and bestatin, when it was preliminary analysed by electrospray ionization mass spectrometry
(ESI-MS) (Fig. 2). 5-FU, bestain and BC-02 were dissolved in dimethyl sulfoxide (DMSO; Sigma-Aldrich, St. Louis, MO, USA) at $400 \mathrm{mM}$ as stock solution. The 5-FU and bestatin was mingled by equimolar concentration at $800 \mathrm{mM}$ as stock solution, it is represented by $1: 1$ in the subsequent experiments. The CD13 mouse anti-human antibody was obtained from Santa Cruz Biotechnology (Santa Cruz, CA, USA). Methyl thiazolyl tetrazolium (MTT) was purchased from Beijing Solarbio Science and Technology Co., Ltd. (Beijing, China).

Cell culture. Human liver cancer cell lines PLC/PRF/5 and Huh7 were purchased from the Cell Bank of Shanghai (Shanghai, China) and cultured in modified Eagle's medium (MEM) and Dulbecco's modified Eagle's medium (DMEM; Life Techologies Corp., Carlsbad, CA, USA) respectively, both supplemented with $10 \%$ fetal bovine serum (FBS; Biological Industries, Kibbutz Beit Haemek, Israel). The cells were cultured at $37^{\circ} \mathrm{C}$ in a humidified $5 \% \mathrm{CO}_{2}$ incubator and passaged every other day.

Sphere culture and self-renewal assay. PLC/PRF/5 and Huh7 parental cells were collected and washed with phosphate buffered saline (PBS) to remove serum, respectively. Then the cells were plated in serum-free stem cell conditional medium composed of DMEM/F12 (HyClone Laboratories, Inc., South Logan, UT, USA), KnockOut ${ }^{\mathrm{TM}}$ serum replacement, 2\% B-27 supplement, $1 \%$ N-2 supplement (Gibco/Invitrogen, Grand Island, NY, USA), $20 \mathrm{ng} / \mathrm{ml}$ recombinant human insulin-like growth factor-I (IGF-I), $20 \mathrm{ng} / \mathrm{ml}$ animal-free recombinant human epidermal growth factor (EGF), $10 \mathrm{ng} / \mathrm{ml}$ recombinant human fibroblast growth factor-basic (b-FGF), (PeproTech, Rocky Hill, NJ, USA), 2 mM L-glutamine, $5 \mu \mathrm{g} / \mathrm{ml}$ heparin sodium and $1 \%$ penicillin-streptomycin (Beijing Solarbio Science and Technology). The cells were suspended in ultralow attachment 6-well plates (Corning Inc., Corning, NY, USA) at a density of 10,000 cells/well. When the spheres grew to $100-200 \mu \mathrm{m}$ in diameter, the spheres were dissociated with trypsin-EDTA. Then the single cells were re-suspended in stem cell conditional medium to re-form spheres and the medium was changed every other day. The PLC/PRF/5 spheres were passaged every 5-7 days. The Huh7 spheres were passaged every 3-5 days. In addition, the isolated PLC/PRF/5 parental cells were cultured in ultralow attachment 6 -well plates at a density of 2,000 cells/well for $48 \mathrm{~h}$. Then cells were treated with $400 \mu \mathrm{M}$ various chemicals (5-FU, bestatin, 1:1, BC-02, and BC-02 + NAC). Cells were pretreated by $500 \mu \mathrm{M}$ $\mathrm{NAC}$ for $2 \mathrm{~h}$. Chemicals were removed after $6 \mathrm{~h}$. After further culturing for 3 days, the spheres were analyzed by optical microscopy.

Colony formation assay. PLC/PRF/5 parental and sphere cells were dissociated into single cells and the cells were seeded in MEM with $10 \%$ FBS on 6-well plates (1,000 cells/well). After 8-10 days, the cells grew to visible colonies ( $>50$ cells). The colonies were counted under an ordinary optical microscope after crystal violet staining. To analyze the effects of chemicals on colony forming of PLC/PRF/5 and Huh7 sphere cells, single cells were cultured in 6-well plates at a density of 10,000 cells/well for $24 \mathrm{~h}$. The cells were treated with various chemicals (5-FU, bestain, 1:1, BC-02 and BC-02 + NAC) 


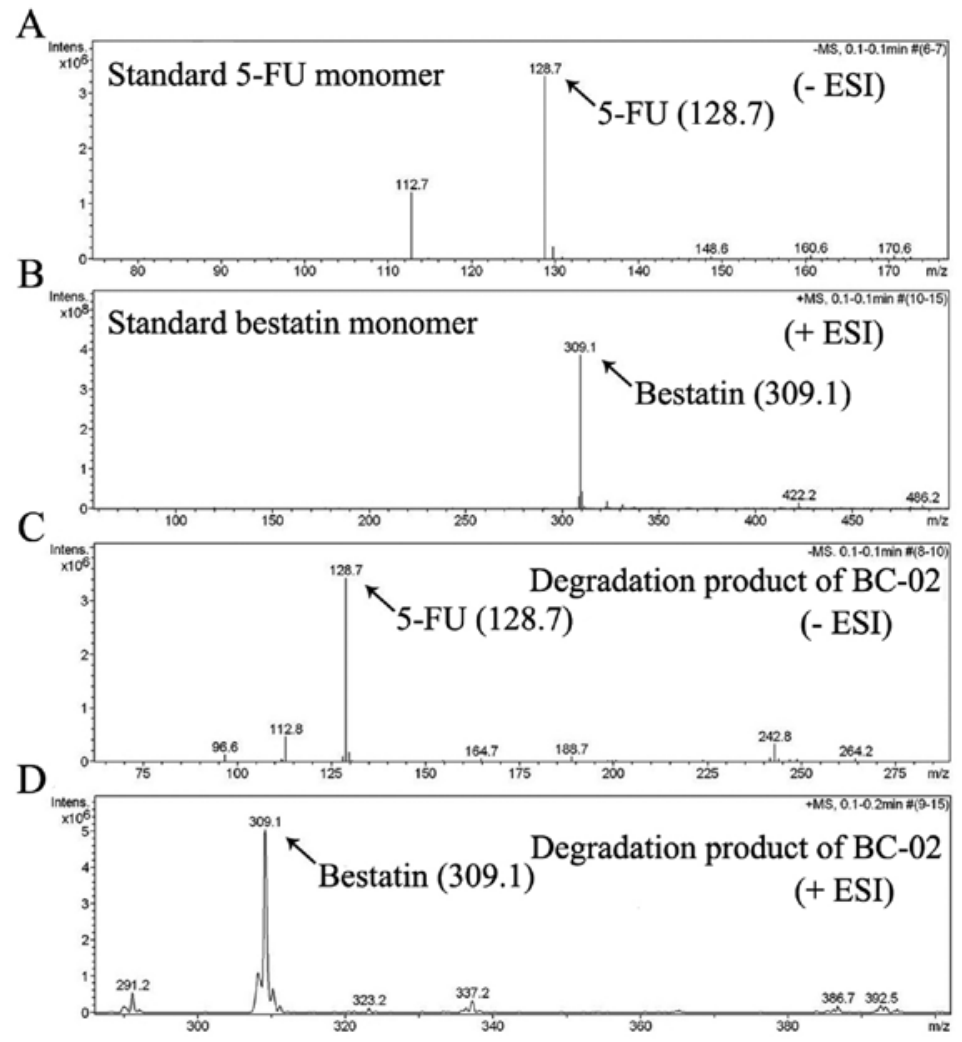

Figure 2. ESI-MS. (A) Standard 5-FU monomer. (B) Standard bestatin monomer. (C and D) Degradation products of BC-02.

for $6 \mathrm{~h}$. Cells were pretreated by $500 \mu \mathrm{M}$ NAC for $2 \mathrm{~h}$. Then cell medium was changed to remove chemicals. The colonies were counted as mentioned and PLC/PRF/5 sphere cells were treated with $400 \mu \mathrm{M}$ drugs, and the concentration of chemicals for Huh7 sphere cells was $200 \mu \mathrm{M}$.

Western blot analysis. Parental and sphere cells were collected, washed with PBS, and lysed in RIPA buffer with protease inhibitor cocktail (Sigma-Aldrich). Protein concentrations were measured using a BCA protein assay kit (Beijing Solarbio Science and Technology). Then quantified proteins were electrophoresed, separated in SDS-polyacrylamide gel (SDS-PAGE) and transferred onto polyvinylidene difluoride (PVDF) membrane (Millipore). Rabbit anti-human N-cadherin antibodies (dilution 1:1,000; Abcam, Cambridge, MA, USA), rabbit anti-human vimentin (dilution 1:1,000; Cell Signaling Technology, Danvers, MA, USA), and mouse anti-human beta actin (dilution 1:1,000; PeproTech) were used as the primary antibodies, and horseradish peroxidase-conjugated anti-rabbit and anti-mouse immunoglobulin (dilution 1:5,000; Beyotime Institute of Biotechnology, Shanghai, China) were used as the secondary antibody. Blots were acquired by enhanced chemiluminescence kit (Millipore).

MTT assays. Cell viability was measured by the MTT assay. $\mathrm{PLC} / \mathrm{PRF} / 5$ parental and sphere cells were dissociated into single cells. The dissociated parental cells were re-suspended in MEM with $10 \%$ FBS on 96-well plates at a density of 5,000 cells/well, and the dispersed sphere cells were re-suspended in serum-free stem cell conditional medium on ultralow attachment 96-well plates (5,000 cells/well). After $24 \mathrm{~h}$, the cells were treated with various concentrations of compounds. Two days after treatment, cells were incubated with MTT solutions for $4 \mathrm{~h}$ at $37^{\circ} \mathrm{C}$. Then the DMSO was added to each well. After that, the absorbance was measured at $570 \mathrm{~nm}$ using a multifunctional microplate reader (SpectraMax M5; Molecular Devices, Sunnyvale, CA, USA). Huh7 cells were treated with the same method. The effects of CD13 inhibition on cell proliferation of spheres were examined using this method.

Cell proliferation in vitro was also measured by MTT method. The enzymatically dissociated cells were seeded in ultralow attachment 96-well plates in $200 \mu \mathrm{l}$ of serum-free stem cell conditional medium at a density of 1,000 cells/well. Cells were fed with stem cell conditional medium every day. The absorbance were measured every $12 \mathrm{~h}$ for 6 consecutive days at $570 \mathrm{~nm}$ using a multifunctional microplate reader and the growth curves were draw using the data.

Enzyme activity assay. The APN activity was determined as previously described (24). PLC/PRF/5 cells were re-suspended in PBS in a 96-well plate with specified concentrations of bestatin or BC-02. Cells were incubated with L-leucine-pnitroanilide (Sigma-Aldrich) for $30 \mathrm{~min}$ at $37^{\circ} \mathrm{C}$. Then the enzyme activity was obtained by measuring the absorbance at $405 \mathrm{~nm}$ using a multifunctional microplate reader. The APN enzyme activity inhibition rates were calculated by (OD control - OD tested)/OD control x 100\%.

Cellular ROS detection. Parental and sphere cells were trypsinized with trypsin-EDTA and the isolated cells were incubated at $37^{\circ} \mathrm{C}$ for $30 \mathrm{~min}$ in darkness with $2^{\prime}, 7^{\prime}$-dichlo- 


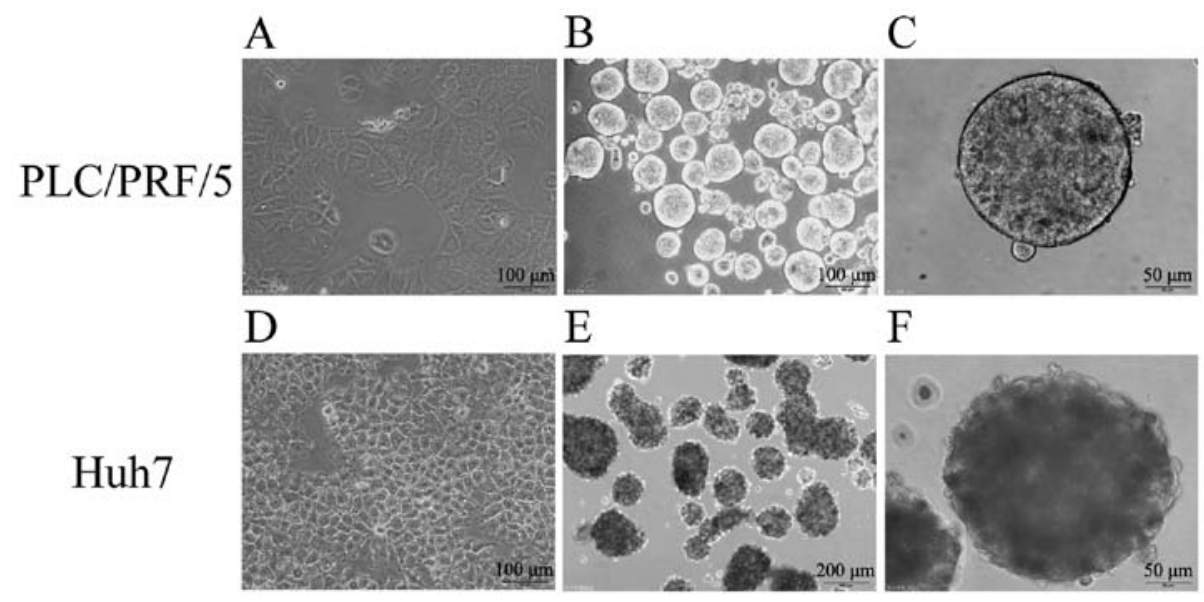

Figure 3. Spheres formed by two hepatoma cell lines (PLC/PRF/5 and Huh7). Hepatoma cell lines PLC/PRF/5 (A) and Huh7 (D) under conventional medium formed epithelial morphology. Cells cultured in stem cell conditional medium acquired sphere morphology (B,C,E and F).

rofluorescein diacetate (DCFH-DA). After washing with PBS, the fluorescence intensity of DCF inside the cells was measured using a FACSCan flow cytometer. Intracellular ROS levels were analyzed using FlowJo 7.6 software and the data represented the mean fluorescence intensity of 30,000 events.

Alkaline comet assay. To determine the oxidative DNA damage, the alkaline comet assay was performed according to the manufacturer's instructions. Briefly, PLC/PRF/5 sphere cells were treated with various drugs $(400 \mu \mathrm{M}$ of $5-\mathrm{FU}$, bestatin, 1:1, BC-02 and BC-02 + NAC) for $12 \mathrm{~h}$. Cells were pretreated by $500 \mu \mathrm{M} \mathrm{NAC}$ for $2 \mathrm{~h}$. Then the cells were dissociated into single cells. The resulting cells were mixed with $0.7 \%$ low-melting agarose, added to slides pre-coated with $0.55 \%$ normal-melting agarose, covered with coverslips and allowed to solidify at $4^{\circ} \mathrm{C}$ for $30 \mathrm{~min}$. Then the coverslips were gently removed and the slides were kept in lysis buffer (2.5 M NaCl, $0.1 \mathrm{M} \mathrm{Na}_{2}$ EDTA, $10 \mathrm{mM}$ Tris, $1 \%$ Triton $\mathrm{X}-100$, and $10 \%$ DMSO, $\mathrm{pH} 10.0$ ) for $1.5 \mathrm{~h}$, submerged in alkaline electrophoresis solution (10 M NaOH, 0.2 M EDTA, $\mathrm{pH}>13$ ) for $50 \mathrm{~min}$, and subjected to electrophoresis in the same buffer at $25 \mathrm{~V}$ for $25 \mathrm{~min}$. After electrophoresis, cells were washed with neutral buffer ( $\mathrm{pH}$ 7.5) and stained with propidium iodide (PI) for $10 \mathrm{~min}$. The images were taken with a fluorescent microscope and the comets were quantified by determination of the percentage of DNA in the tail using TriTek Comet Score ${ }^{\mathrm{TM}}$ Freeware v1.5. Huh7 cells were treated with the same method and the concentration of the chemicals was $200 \mu \mathrm{M}$.

Nude mouse tumor formation assay. Four to five week-old female nude mice were purchased from the Hunan SJA Laboratory Animal Co., Ltd.,2 (Hunan, China) and maintained in a specific pathogen-free conditions. All animal experiments were approved by the Guidelines of the Animal Care and Use Committee at Weifang Medical University. The defined number of viable parental and sphere cells was subcutaneously injected into the left or right flank of mice. After the inoculation, tumor formation and growth were monitored every day and mice were sacrificed at 12 weeks.
Table I. Comparison of the tumorigenesis of $\mathrm{PLC} / \mathrm{PRF} / 5$ parental cells and sphere cells using nude mice.

\begin{tabular}{lcc}
\hline No. of cells injected & Sphere & Parents \\
\hline $1 \times 10^{5}$ & $1 / 5$ & $0 / 5$ \\
$2 \times 10^{5}$ & $2 / 5$ & $0 / 5$ \\
$1 \times 10^{6}$ & $3 / 5$ & $0 / 5$ \\
\hline
\end{tabular}

Statistical analysis. Each experiment was performed at least three times, and quantitative data were expressed as mean \pm standard deviation (SD). Statistical differences between the two groups were evaluated by the Student's t-test using the SPSS 17.0 statistical software (SPSS, Inc., Chicago, IL, USA). $\mathrm{P}<0.05$ was defined as significant.

\section{Results}

PLC/PRF/5 and Huh7 cells form suspended spheres. Previous studies demonstrated that the spheres, which are suspended culture of cancer cells with growth factors in serum-free medium, are enriched with CSCs. To successfully induct CSCs, a more suitable culture system was first determined. Through careful screening, the suitable culture, which contains DMEM/F12, serum replacement, B-27 supplement, N-2 supplement, IGF-I, EGF, b-FGF, L-glutamine, heparin sodium and penicillin-streptomycin, was determined. In the culture system, two human HCC cell lines, namely, PLC/ PRF/5 and Huh7, successfully formed suspended spheres. The status of the spheres was stably maintained even after several generations, indicating their capability for self-renewal similarly to TICs. Fig. 3 shows that cells under conventional medium with 10\% FBS formed epithelial morphology, and the images of cells cultured in the serum-free stem cell conditional medium formed suspended, self-renewing spheres, which were observed by a microscope.

PLC/PRF/5 and Huh7 sphere cells possess the characteristics of CSCs. Both PLC/PRF/5 and Huh7 spheres possessed the characteristics of CSCs, including drug resistance, high 
A

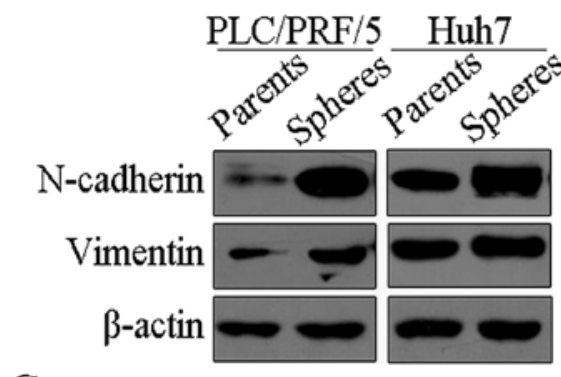

C

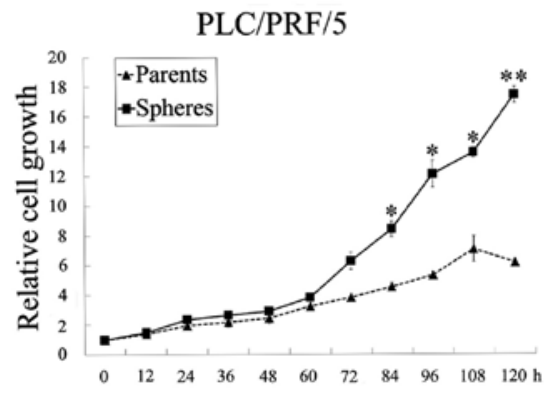

E

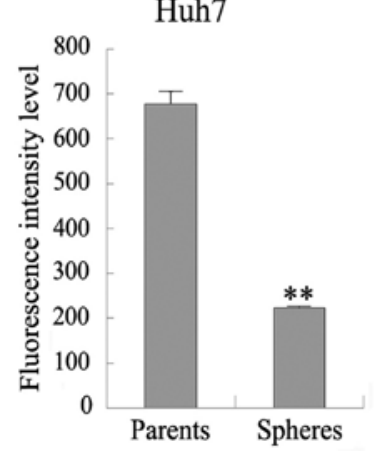

B

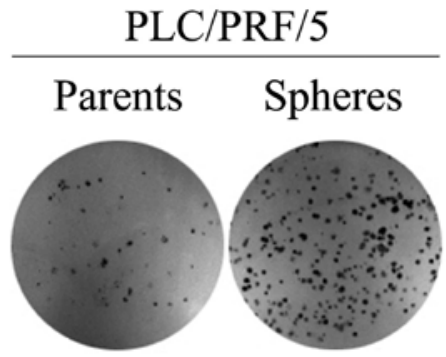

D

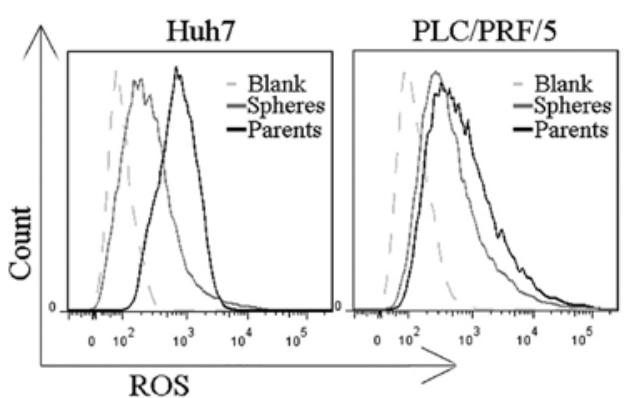

F

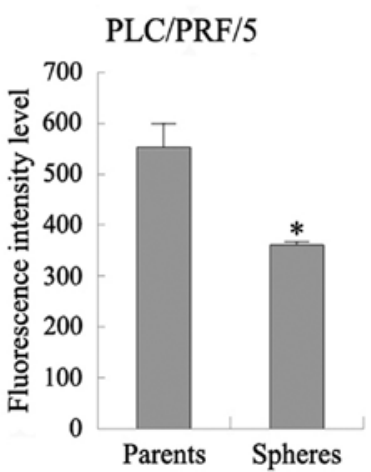

Figure 4. Characterization of CSCs in PLC/PRF/5 and Huh7 spheres. (A) The epithelial-mesenchymal transition (EMT) properties were analyzed by western blot analysis. Sphere cells highly expressed mesenchymal cell biomarker N-cadherin and vimentin. (B) The clonogenic ability of the PLC/PRF/5 sphere cells and parental cells was examined. Sphere cells formed greater number of colonies than their parental cells. Representative photographs are provided. (C) The proliferation of the $\mathrm{PLC} / \mathrm{PRF} / 5$ sphere cells was faster than their parental cells in vitro. Experiments were performed in triplicate $\left({ }^{*} \mathrm{P}<0.05\right.$, $\left.{ }^{* *} \mathrm{P}<0.01\right)$. (D) Intracellular ROS concentrations stained with DCFH-DA were measured using a FACSCan flow cytometer. (E) The mean fluorescence intensity was lower in Huh7 sphere cells than in their parental cells. Three independent experiments were performed and the data represent the mean $\pm \mathrm{SD}\left({ }^{* *} \mathrm{P}<0.01\right)$. (F) The mean fluorescence intensity was lower in PLC/PRF/5 sphere cells than in their parental cells ( $\mathrm{P}<0.05)$.

tumorigenicity, EMT phenotype, lower ROS levels, greater colony-forming efficiency, and increased proliferation capacity in vitro. MTT assays were performed to examine the sensitivity of spheres and parental cells to our chemotherapy drugs. Spheres and parental cells of liver cancer cell lines, such as PLC/PRF/5 and Huh7, were treated with 5-FU, bestatin, 1:1, and BC-02, respectively, with different concentrations for $48 \mathrm{~h}$. As shown in Fig. 5A and B, the spheres showed much lower inhibition effect of the chemotherapy drugs than their parental cells, which indicates that spheres had increased chemoresistance. Tumorigenicity in vivo was analyzed by tumor formation assay using nude mice. As shown in Table I, $1 \times 10^{5}$ sphere cells derived from PLC/PRF/5 cells successfully formed tumors in one out of five mice, whereas similar number of PLC/PRF/5 parental cells failed to form any tumors. Furthermore, although the number of parental cells increased to $1 \times 10^{6}$, there was no tumor generated. In addition, $1 \times 10^{6}$ sphere cells were capable of forming tumors in three out of five mice. This result indicated that the sphere cells possessed high tumorigenicity in vivo and increased CSC properties. Recently, EMT has been considered to be associated with CSC stemness characteristic. EMT-related regulatory proteins, including $\mathrm{N}$-cadherin and vimentin, were examined by western blotting. As shown in Fig. 4A, the mesenchymal markers (N-cadherin and vimentin) were upregulated in PLC/PRF/5 and Huh7 spheres compared with their parental cancer cells. Cancer stem cells which are similar to stem cells normally possess low levels of intracellular ROS. Thus, flow cytometric analyses were performed to examine intracellular ROS levels. Intracellular ROS levels both in PLC/PRF/5 and in HuH7 were lower in sphere cells than in parental cells (Fig. 4D-F). Colony formation and cell proliferation assays were also performed to evaluate the proliferative ability in vitro of the PLC/PRF/5 sphere and parental cells. As shown in Fig. 4B, more clone numbers of the sphere cells were significantly formed compared with the parental cells, and the proliferation of the PLC/PRF/5 sphere cells was 



Drug concentration $(\mu \mathrm{M})$

F 120

$\mathrm{PLC} / \mathrm{PRF} / 5$

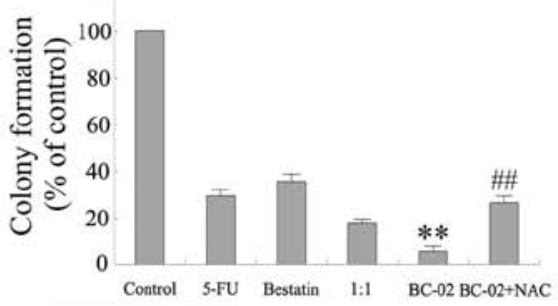

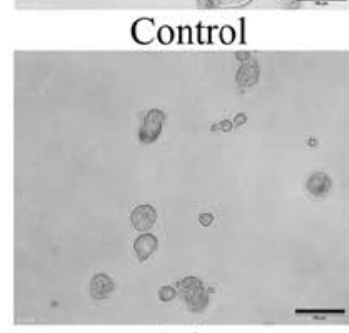

$1: 1$

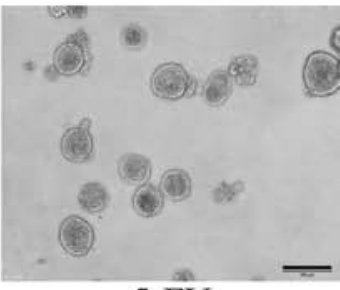

5-FU

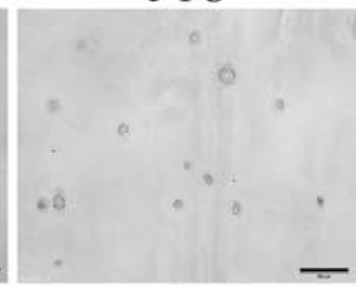

BC-02

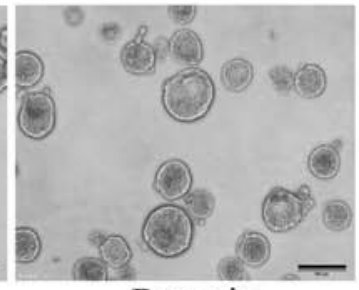

Bestatin

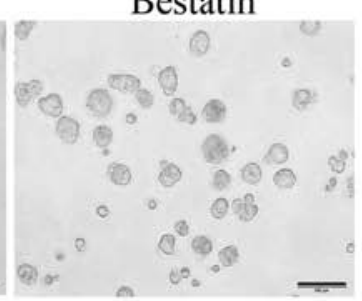

$\mathrm{BC}-02+\mathrm{NAC}$

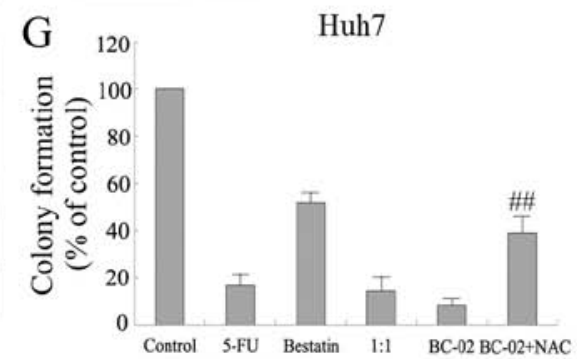

Figure 5. Effects of BC-02 on self-renewal and proliferation of sphere cells. (A) PLC/PRF/5 cell line. (B) Huh7 cell line. Spheres and parental cells were exposed to various concentrations of chemotherapy drugs for $48 \mathrm{~h}$. Cell vitality was determined by the MTT assay. Bars represent the mean of three determinations \pm SD. (C) Sphere formation assay of PLC/PRF/5 cell line was performed. Sphere cells were cultured in the absence or presence of $400 \mu \mathrm{M} \mathrm{different}$ chemotherapy drugs for $6 \mathrm{~h}$. BC-02 markedly inhibited the number and size of PLC/PRF/5 spheres. Bright-field images are shown. Scale bar, $100 \mu \mathrm{m}$. (D) PLC/PRF/5. (E) Huh7. The clonogenic ability of sphere cells stimulated with various agents was examined using colony formation assay. Representative examples are provided. (F) Statistical analysis of (D). Three independent experiments were performed and the data represent the mean \pm SD $\left({ }^{* *} \mathrm{P}<0.01 \mathrm{vs} .5\right.$-FU or $1: 1,{ }^{\# \#} \mathrm{P}<0.01$ vs. BC-02). (G) Statistical analysis of (E). Three independent experiments were performed and the data represent the mean \pm SD $\left({ }^{\# \#} \mathrm{P}<0.01 \mathrm{vs}\right.$. BC-02).

faster than that of their parental cells (Fig. 4C). These results supported the greater proliferative ability in vitro.

BC-02 effectively inhibits proliferation and self-renewal of liver cancer stem cell. Spheres cultured in stem cell conditional medium have been proven to possess the characteristics of CSCs and to enrich the CSCs. Then, the effect of BC-02 on $\mathrm{PLC} / \mathrm{PRF} / 5$ or Huh7 spheres in vitro assays was examined. Both cell lines, namely, PLC/PRF/5 and Huh7, were used to perform cell proliferation assay. BC-02 significantly suppressed the proliferation not only of parental cells but also sphere cells compared with other chemotherapy drugs in a dose-dependent manner (Fig. 5A and B). Furthermore, sphere formation experiments of PLC/PRF/5 cell line were performed using different chemotherapy drugs, such as 5-FU, bestatin, 1:1 and BC-02, with similar concentrations. As shown in Fig. 5C, BC-02 markedly inhibited the number and size of tumor spheres compared with the control, bestatin, 5-FU and even 1:1 groups. In addition, BC-02 also effectively impaired the formation of colonies compared with other groups not only of PLC/PRF/5 sphere cells but also of Huh7 sphere cells (Fig. 5D-G). Overall, these results indicate that 

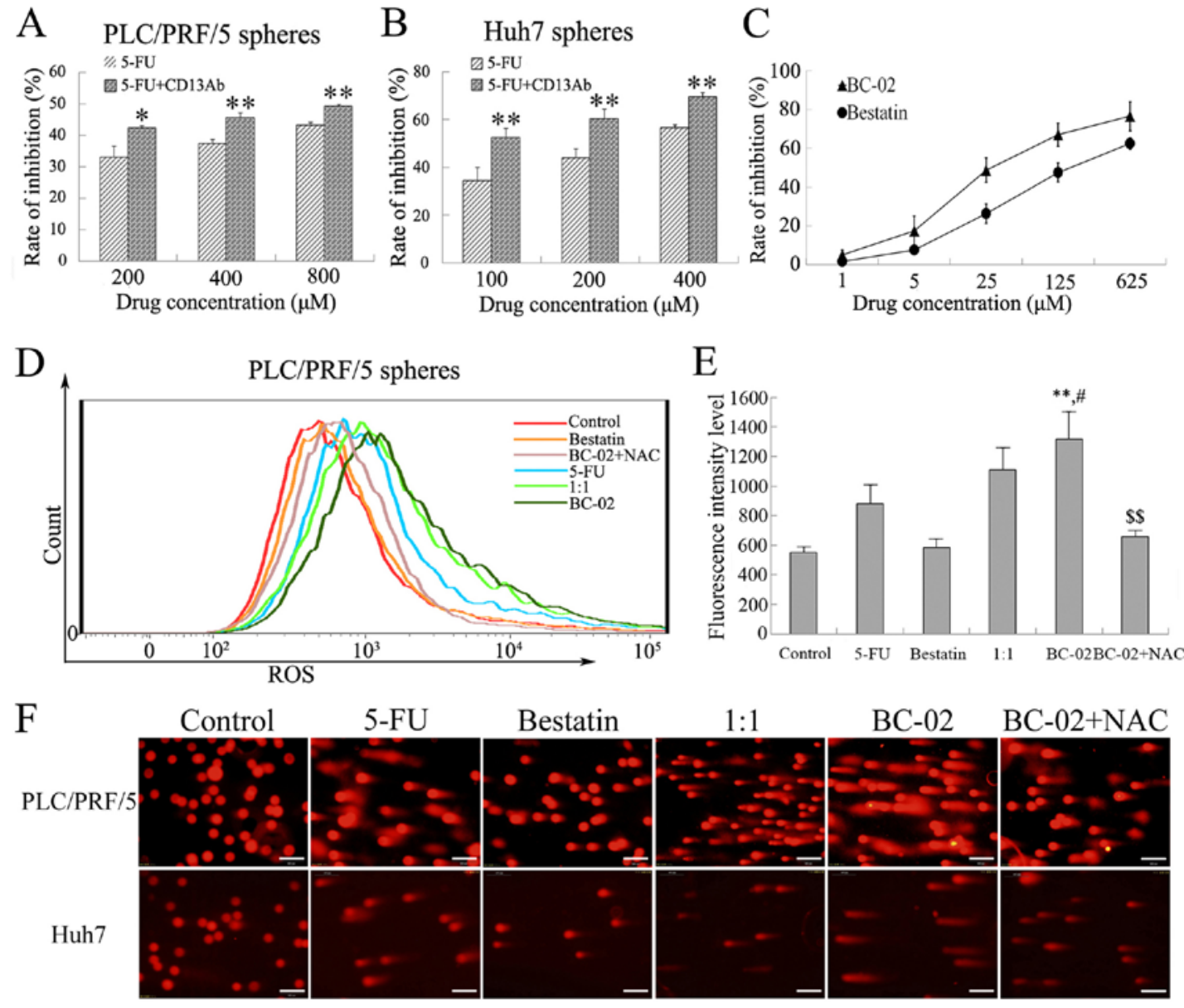

$\mathrm{G}$
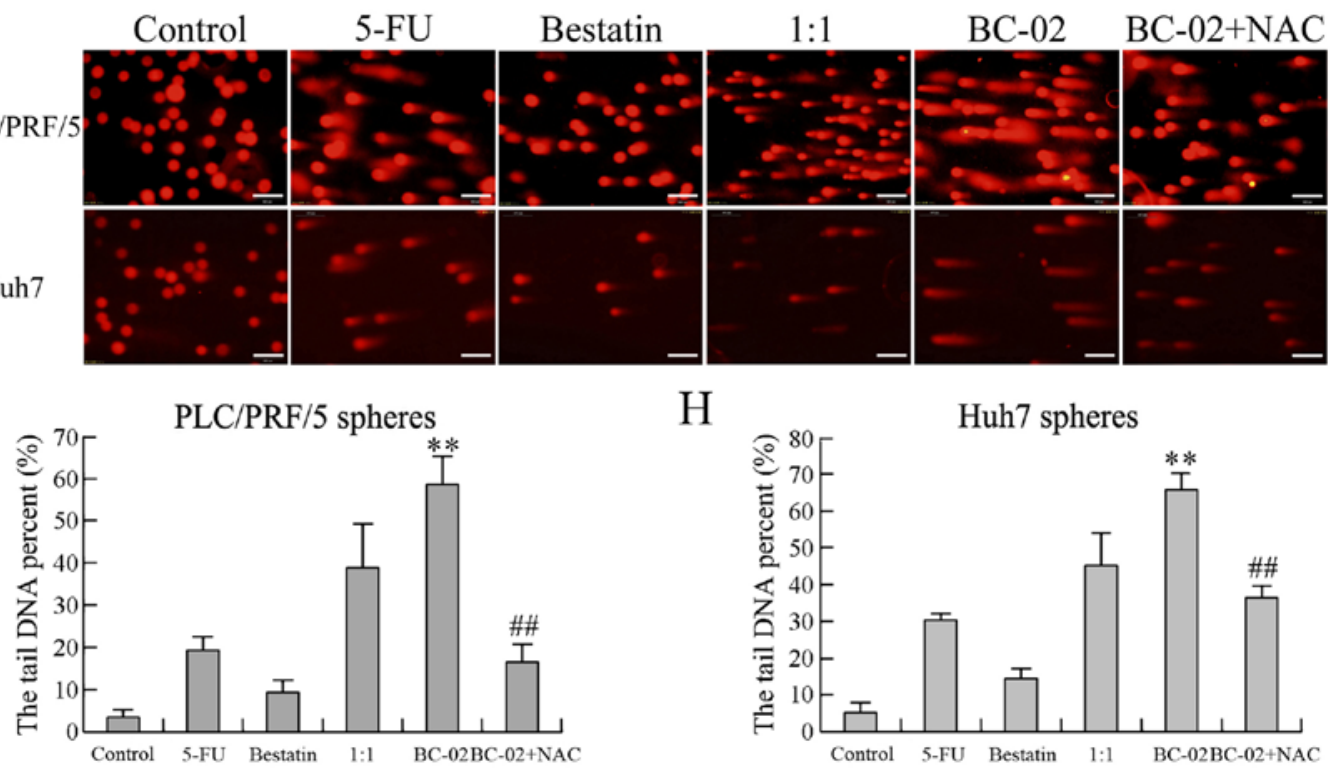

$\mathrm{H}$

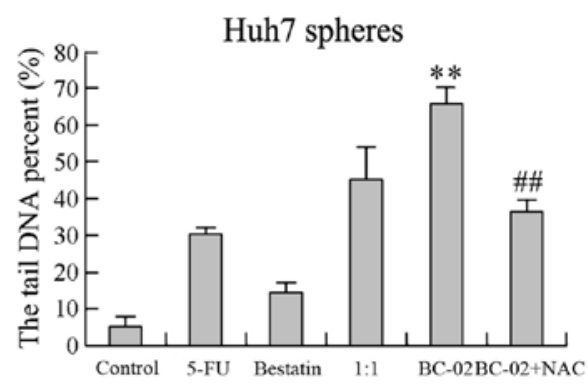

Figure 6. Effects of BC-02 on activity of CD13 and DNA damage dependent on ROS. (A) PLC/PRF/5 spheres. (B) Huh7 spheres. Effects of CD13 inhibition on cell proliferation of PLC/PRF/5 spheres and Huh7 spheres. The spheres were cultured with 5-FU or 5-FU plus $2 \mu \mathrm{g} / \mathrm{ml}$ CD13 Ab for $48 \mathrm{~h}$. Data represent the mean $\pm \mathrm{SD}\left({ }^{*} \mathrm{P}<0.05,{ }^{* *} \mathrm{P}<0.01\right)$. (C) Inhibitory effects of BC-02 or bestatin on activity of CD13 in PLC/PRF/5 cells. (D) PLC/PRF/5 sphere cells were exposed to $200 \mu \mathrm{M}$ of drug samples for $15 \mathrm{~h}$. Intracellular ROS concentrations were measured using a FACSCan flow cytometer. (E) Statistical analysis of the mean fluorescence intensity. Three independent experiments were performed and the data represent the mean $\pm \mathrm{SD}\left({ }^{* *} \mathrm{P}<0.01\right.$ vs. control, ${ }^{\#} \mathrm{P}<0.05$ vs. 5 -FU, ${ }^{\$ \$} \mathrm{P}<0.01$ vs. BC-02). (F) Sphere cells incubated with the indicated drugs for $12 \mathrm{~h}$ were analyzed in an alkaline comet assay to measure DNA damage. Representative images are shown. Scale bar, $200 \mu \mathrm{m}$. Statistical analysis of DNA strand breaks by measuring the percent of tail-DNA in (G) PLC/PRF/5 spheres and (H) Huh7 spheres $\left({ }^{* *} \mathrm{P}<0.01\right.$ vs. 5 -FU or $1: 1,{ }^{\# \#} \mathrm{P}<0.01$ vs. $\left.\mathrm{BC}-02\right)$.

BC-02 effectively suppresses malignant proliferation and self-renewal of CSCs. The co-treatment of sphere cells with the ROS scavenger NAC, which is an antioxidant, attenuated the inhibitory effect on proliferation, self-renewal and clone formation induced by BC-02. Those results prompted that lower level of ROS may be a protection for cells and BC-02 may damage the cells by increasing the intracellular ROS.

BC-02 prevents the properties of hepatoma stem cells by targeting CD13 and increasing the ROS-dependent DNA damage. CD13 is a therapeutic target in human LCSCs. The effect of CD13 inhibition on cell proliferation was confirmed by cell proliferation assay in PLC/PRF/5 and Huh7 sphere cells. Cell proliferation was effectively suppressed after exposure to 5-FU plus CD13 Ab compared with 5-FU alone (Fig. 6A and B). In addition, bestatin was recognized as CD13 inhibitor, which specifically blocks CD13 and antagonizes with the zinc-binding site of the CD13 domain. The inhibitory effects of bestatin and BC-02 on CD13 activity were measured using PLC/PRF/5 cells, which had high expression levels of CD13. As shown in Fig. 6C, CD13 activity was inhibited by bestatin as reported. BC-02 even showed higher inhibition rate than bestatin in a concentration-dependent manner. These results indicated that $\mathrm{BC}-02$ was more effective to target CD13 


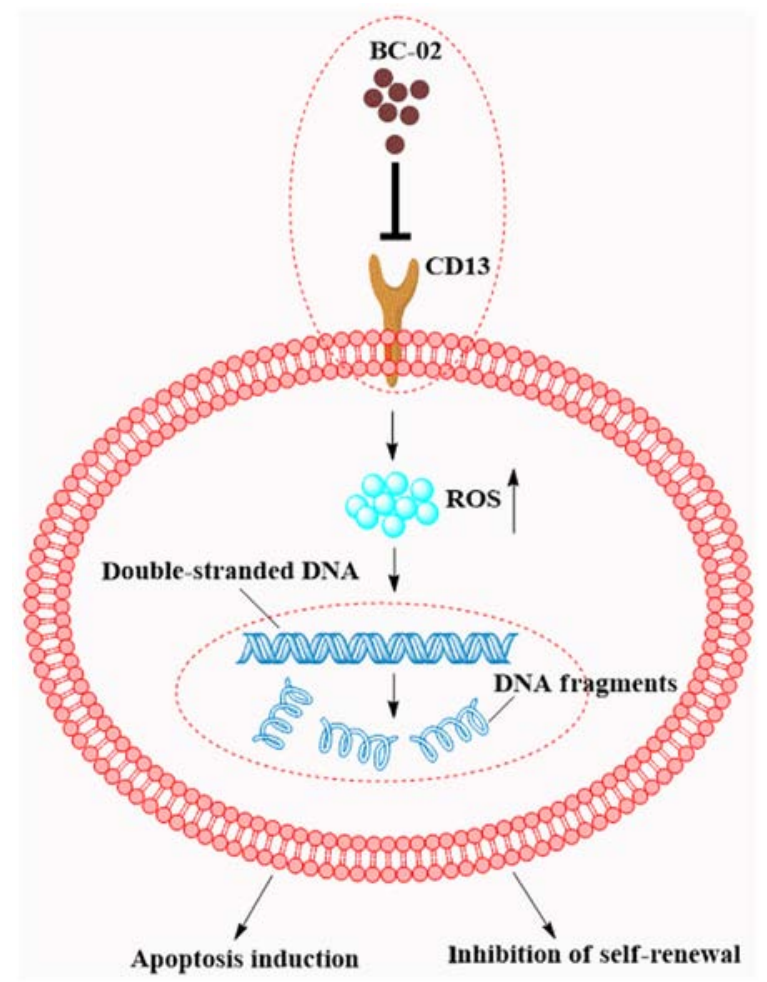

Figure 7. The mechanism behind the effects of BC-02 on the liver CSC proliferation. BC-02 could target CD13 and cause an increase in intracellular ROS and ROS-induced DNA damage.

than bestatin; thus, inhibition of CD13 by BC-02 contributes to the inhibition of CSCs.

To investigate further the mechanism by which BC-02 suppresses self-renewal and malignant proliferation of CSCs, the intracellular ROS levels were detected by prooxidants using the fluorescence dye DCF-DA. In addition, ROS-induced DNA damage after genotoxic chemo-stress was detected by an alkaline comet assay. As mentioned, ROS played an important role in CSC proliferation. The intracellular ROS levels were evaluated when cells were exposed to different chemotherapeutic drugs $(200 \mu \mathrm{M}$ of 5-FU, bestatin, 1:1, BC-02 and BC-02 + NAC) for $15 \mathrm{~h}$. A significant increase in ROS level was observed in PLC/PRF/5 sphere cells treated with BC-02 compared with control, bestatin, 5-FU, and even 1:1 group (Fig. 6D and E), and the co-treatment with NAC canceled the level of ROS induced by BC-02. In addition, the most significant DNA damage with the longest cometic tail of DNA in both PLC/PRF/5 and Huh7 sphere cells was induced by $\mathrm{BC}-02$ compared with other groups. The DNA damage was significantly reduced when NAC was co-treated with BC-02 (Fig. 6F-H). Overall, BC-02 targeted CD13 and caused an increase in intracellular ROS and ROS-induced DNA damage in hepatoma stem cells.

\section{Discussion}

CSCs with stem cell properties are a minor population of cancer cells. Their presence is responsible for the chemoresistance to the systemic chemotherapy in HCC. Therefore, searching for a novel agent that can target CSCs and exhibit cytotoxic effects on CSCs for eradicating liver CSCs and improving the prognosis of patients with $\mathrm{HCC}$ were indispensable. In this study, BC-02 can potently inhibit the self-renewal and proliferation of liver CSCs by targeting CD13 and increasing the intracellular ROS and the ROS-induced DNA damage.

PLC/PRF/5 and Huh7 spheres formed and acquired stemness in the serum-free medium. The sphere cells had several characteristics of CSCs, including drug resistance, high tumorigenicity in nude mice, EMT phenotype, lower ROS levels, greater colony-forming efficiency, and increased proliferation capacity in vitro. Similar to previous studies, spheres possessed the stemness that was expanded by sphere culture methods (27-30). What was interesting was that the proliferation of the Huh7 sphere cells was slower than their parental cells (data not shown), which may be because the culture medium was not completely appropriate to the Huh7 cell line, and the cells could not enter a rapid proliferation period. Thus, different CSCs had different microenvironments that should be adaptive to their survival. Efforts are still necessary for exploring more suitable CSC media for various cell lines.

5 -FU is one of the most common anticancer drugs in HCC treatment. It has toxicity to the hematopoietic function of bone marrow $(31,32)$. Bestatin is a recognized CD13 inhibitor that is constantly used as an adjuvant, which can enhance the immunity and prolong the lifetime of patients with acute adult non-lymphocytic leukemia. Studies have reported that the combination therapy of 5-FU plus bestatin efficiently enhanced the antitumor effects of liver cancer $(5,33)$. In this study, BC-02 was synthesized with the 5-FU and bestatin monomer. BC-02 could target CD13 and enter the cells accurately with cytotoxic effects. Thus, it might reduce the drug side-effects by cutting down the dosage and increasing the efficacy of chemotherapeutics. BC-02 can effectively inhibit the activity of CD13 compared with bestatin in a dose-dependent manner. Therefore, $\mathrm{BC}-02$ is a potential molecular targeting agent to CD13. BC-02 also can slowly release bestatin and 5-FU (Fig. 2). Thus, BC-02 which is similar to 5-FU can possibly exploit the advantages of cytotoxicity to cancer cells. As expected, BC-02 effectively suppressed the number and size of tumor spheres, the formation of colonies, and the malignant proliferation of the CSCs compared with 5-FU, bestatin, and even the combination 5-FU with bestatin. In brief, the newfangled CD13 inhibitor BC-02 could efficiently inhibit self-renewal and proliferation of liver CSCs. Experiments in vivo must be conducted for further research on this novel antineoplastic agent.

Previous findings indicated that CSCs could always resist chemotherapy drugs and maintain long-term survival. Evidence showed that those characters of CSCs are related to cell cycle dormancy, lowering the ROS levels in CSCs, and DNA repair mechanisms (34). In the present study, the spheres of PLC/PRF/5 and Huh7 contained lower intracellular ROS levels than their parental cells. Previous studies suggested that chemotherapeutic drugs were used to cause DNA damage through ROS accumulation (35), and the combination therapy of 5-FU plus bestatin efficiently enhanced the antitumor effects by upregulating intracellular ROS levels and inducing DNA injury $(5,33)$. Whether generating similar processes of liver CSCs after exposure to BC-02 was examined. As expected, BC-02 effectively inhibited self-renewal and proliferation of CSCs, increased the intracellular ROS and promoted the 
DNA oxidative damage. The treatment of CSCs with NAC recovered the proliferation of CSCs, canceled the increase of the ROS level and repaired the DNA damage. The molecular mechanism of BC-02 to CSCs was consistent with previous studies. However, further studies are required to determine the molecular mechanism of increasing production of intracellular ROS or reducing the scavenger of ROS when CSCs are exposed to $\mathrm{BC}-02$.

A recent series of studies provided further evidence that cancer progress is dependent not only on the malignant cells but also on the microenvironment. This study demonstrated that glioblastoma, one of the malignant brain tumors, maintains their CSCs in vascular niches. Targeting cancer stem cell niches may be a powerful approach for the treatment of cancer (36). Recent data showed that anti-angiogenic strategies combined with conventional cytotoxic drugs reduce the tumor stem-like cell fraction (37). Sorafenib is always used in the systemic treatment of HCC, and it is the inhibitor the receptors of the anti-angiogenic multi-kinase. Thus, BC-02 combined with sorafenib might effectively provide curative effect for patients with HCC.

In conclusion, we indicated that combination of BC-02 may efficiently inhibit the self-renewal and proliferation of liver CSCs compared with 5-FU, bestatin, and even the combination of 5-FU and bestatin. Furthermore, as summarized in Fig. 7, BC-02 was able to target CD13 and then increase the intracellular ROS and the ROS-induced DNA damage. This will lead to the inhibition of self-renewal and induction of apoptosis of LCSCs. Therefore, the novel CD13 inhibitor BC-02 may be a potential strategy for eradicating liver CSCs and overcoming chemoresistance in liver cancer.

\section{Acknowledgements}

The present study was supported by the National Natural Science Foundation of China (no. 81503108), the Project of Shandong Province Higher Educational Science and Technology Program (J15LM58), the Research Award Fund for Outstanding Young and Middle-aged Scientists of Shandong Province (no. BS2015YY016), and the National Natural Science Foundation of China (nos. 81373282, 81201262 and 81471048).

\section{References}

1. Mlynarsky L, Menachem Y and Shibolet O: Treatment of hepatocellular carcinoma: Steps forward but still a long way to go. World J Hepatol 7: 566-574, 2015.

2. Siegel R, Ma J, Zou Z and Jemal A: Cancer statistics, 2014. CA Cancer J Clin 64: 9-29, 2014.

3. Wei KR, Yu X, Zheng RS, Peng XB, Zhang SW, Ji MF, Liang ZH, Ou ZX and Chen WQ: Incidence and mortality of liver cancer in China, 2010. Chin J Cancer 33: 388-394, 2014.

4. DeSantis CE, Lin CC, Mariotto AB, Siegel RL, Stein KD, Kramer JL, Alteri R, Robbins AS and Jemal A: Cancer treatment and survivorship statistics, 2014. CA Cancer J Clin 64: 252-271, 2014.

5. Haraguchi N, Ishii H, Mimori K, Tanaka F, Ohkuma M, Kim HM, Akita H, Takiuchi D, Hatano H, Nagano H, et al: CD13 is a therapeutic target in human liver cancer stem cells. J Clin Invest 120: 3326-3339, 2010

6. Bruce WR and Van Der Gaag H: A quantitative assay for the number of murine lymphoma cells capable of proliferation in vivo. Nature 199: 79-80, 1963.
7. Bonnet D and Dick JE: Human acute myeloid leukemia is organized as a hierarchy that originates from a primitive hematopoietic cell. Nat Med 3: 730-737, 1997.

8. Visvader JE and Lindeman GJ: Cancer stem cells in solid tumours: Accumulating evidence and unresolved questions. Nat Rev Cancer 8: 755-768, 2008

9. Al-Hajj M, Wicha MS, Benito-Hernandez A, Morrison SJ and Clarke MF: Prospective identification of tumorigenic breast cancer cells. Proc Natl Acad Sci USA 100: 3983-3988, 2003.

10. O'Brien CA, Pollett A, Gallinger S and Dick JE: A human colon cancer cell capable of initiating tumour growth in immunodeficient mice. Nature 445: 106-110, 2007.

11. Singh SK, Hawkins C, Clarke ID, Squire JA, Bayani J, Hide T, Henkelman RM, Cusimano MD and Dirks PB: Identification of human brain tumour initiating cells. Nature 432: 396-401, 2004.

12. Cheung ST, Cheung PF, Cheng CK, Wong NC and Fan ST: Granulin-epithelin precursor and ATP-dependent binding cassette (ABC)B5 regulate liver cancer cell chemoresistance. Gastroenterology 140: 344-355, 2011.

13. Reya T, Morrison SJ, Clarke MF and Weissman IL: Stem cells, cancer, and cancer stem cells. Nature 414: 105-111, 2001.

14. Nguyen LV, Vanner R, Dirks P and Eaves CJ: Cancer stem cells: An evolving concept. Nat Rev Cancer 12: 133-143, 2012.

15. Pang RW and Poon RT: Cancer stem cell as a potential therapeutic target in hepatocellular carcinoma. Curr Cancer Drug Targets 12: 1081-1094, 2012.

16. Yu SC, Ping YF, Yi L, Zhou ZH, Chen JH, Yao XH, Gao L, Wang JM and Bian XW: Isolation and characterization of cancer stem cells from a human glioblastoma cell line U87. Cancer Lett 265: 124-134, 2008.

17. Cao L, Zhou Y, Zhai B, Liao J, Xu W, Zhang R, Li J, Zhang Y, Chen L, Qian H, et al: Sphere-forming cell subpopulations with cancer stem cell properties in human hepatoma cell lines. BMC Gastroenterol 11: 71,2011.

18. Singh SK, Clarke ID, Terasaki M, Bonn VE, Hawkins C, Squire J and Dirks PB: Identification of a cancer stem cell in human brain tumors. Cancer Res 63: 5821-5828, 2003.

19. Pan Z, Hooley J, Smith DH, Young P, Roberts PE and Mather JP: Establishment of human ovarian serous carcinomas cell lines in serum free media. Methods 56: 432-439, 2012.

20. Roberts PE: Isolation and establishment of human tumor stem cells. Methods Cell Biol 86: 325-342, 2008.

21. Ishii K, Usui S, Sugimura Y, Yoshida S, Hioki T, Tatematsu M, Yamamoto $\mathrm{H}$ and Hirano $\mathrm{K}$ : Aminopeptidase $\mathrm{N}$ regulated by zinc in human prostate participates in tumor cell invasion. Int $\mathbf{J}$ Cancer 92: 49-54, 2001.

22. Hashida H, Takabayashi A, Kanai M, Adachi M, Kondo K, Kohno N, Yamaoka Y and Miyake M: Aminopeptidase N is involved in cell motility and angiogenesis: Its clinical significance in human colon cancer. Gastroenterology 122: 376-386, 2002.

23. Tokuhara T, Hattori N, Ishida H, Hirai T, Higashiyama M, Kodama $\mathrm{K}$ and Miyake M: Clinical significance of aminopeptidase $\mathrm{N}$ in non-small cell lung cancer. Clin Cancer Res 12: 3971-3978, 2006.

24. Sun ZP, Zhang J, Shi LH, Zhang XR, Duan Y, Xu WF, Dai G and Wang XJ: Aminopeptidase N inhibitor 4cc synergizes antitumor effects of 5-fluorouracil on human liver cancer cells through ROS-dependent CD13 inhibition. Biomed Pharmacother 76: 65-72, 2015.

25. Kobayashi T, Miyawaki S, Tanimoto M, Kuriyama K, Murakami H, Yoshida M, Minami S, Minato K, Tsubaki K, Ohmoto E, et al; The Japan Leukemia Study Group: Randomized trials between behenoyl cytarabine and cytarabine in combination induction and consolidation therapy, and with or without ubenimex after maintenance/intensification therapy in adult acute myeloid leukemia. J Clin Oncol 14: 204-213, 1996.

26. Jiang Y: School of Pharmacy, Shandong University, PhD Dissertations, Development of novel anti-cancer drugs base on aminopeptidase N/CD13, 2016.

27. Huang YJ and Hsu SH: Acquisition of epithelial-mesenchymal transition and cancer stem-like phenotypes within chitosan-hyaluronan membrane-derived 3D tumor spheroids. Biomaterials 35: 10070-10079, 2014.

28. Han M, Wang Y, Liu M, Bi X, Bao J, Zeng N, Zhu Z, Mo Z, Wu C and Chen X: MiR-21 regulates epithelial-mesenchymal transition phenotype and hypoxia-inducible factor- $1 \alpha$ expression in thirdsphere forming breast cancer stem cell-like cells. Cancer Sci 103: 1058-1064, 2012. 
29. Rybak AP, He L, Kapoor A, Cutz JC and Tang D: Characterization of sphere-propagating cells with stem-like properties from DU145 prostate cancer cells. Biochim Biophys Acta 1813: 683-694, 2011.

30. Qiu X, Wang Z, Li Y, Miao Y, Ren Y and Luan Y: Characterization of sphere-forming cells with stem-like properties from the small cell lung cancer cell line H446. Cancer Lett 323: 161-170, 2012.

31. Di Lorenzo G, Rea A, Carlomagno C, Pepe S, Palmieri G, Labianca R, Chirianni A, De Stefano A, Esposito V, De Placido S, et al: Activity and safety of pegylated liposomal doxorubicin, 5-fluorouracil and folinic acid in inoperable hepatocellular carcinoma: A phase II study. World J Gastroenterol 13: 6553-6557, 2007.

32. Amstutz U, Froehlich TK and Largiadèr CR: Dihydropyrimidine dehydrogenase gene as a major predictor of severe 5-fluorouracil toxicity. Pharmacogenomics 12: 1321-1336, 2011.

33. Yamashita M, Wada H, Eguchi H, Ogawa H, Yamada D, Noda T, Asaoka T, Kawamoto K, Gotoh K, Umeshita K, et al: A CD13 inhibitor, ubenimex, synergistically enhances the effects of anticancer drugs in hepatocellular carcinoma. Int J Oncol 49: 89-98, 2016.
34. Naka K, Muraguchi T, Hoshii T and Hirao A: Regulation of reactive oxygen species and genomic stability in hematopoietic stem cells. Antioxid Redox Signal 10: 1883-1894, 2008.

35. Chetram MA, Bethea DA, Odero-Marah VA, Don-SaluHewage AS, Jones KJ and Hinton CV: ROS-mediated activation of AKT induces apoptosis via pVHL in prostate cancer cells. Mol Cell Biochem 376: 63-71, 2013.

36. Gilbertson RJ and Rich JN: Making a tumour's bed: Glioblastoma stem cells and the vascular niche. Nat Rev Cancer 7: 733-736, 2007.

37. Folkins C, Man S, Xu P, Shaked Y, Hicklin DJ and Kerbel RS: Anticancer therapies combining antiangiogenic and tumor cell cytotoxic effects reduce the tumor stem-like cell fraction in glioma xenograft tumors. Cancer Res 67: 3560-3564, 2007. 